\title{
INFLUENCE OF SUBINHIBITORY ANTIBIOTIC CONCENTRATION ON STREPTOCOCCUS PYOGENES ADHERENCE AND BIOFILM PRODUCTION
}

\author{
ALEKSANDRA ŠMITRAN ${ }^{1 *}$, DRAGANA VUKOVIĆ ${ }^{2}$, NATAŠA OPAVSKI ${ }^{2}$, INA GAJIĆ ${ }^{2}$, \\ JELENA MARINKOVIĆ ${ }^{3}$, LJILJANA BOŽIĆ ${ }^{1}$, IRENA ŽIVANOVIĆ ${ }^{2}$, DUŠAN KEKIĆ ${ }^{2}$, \\ SUNČICA POPOVIĆ ${ }^{2}$ and LAZAR RANIN ${ }^{2}$ \\ ${ }^{1}$ Faculty of Medicine, Department of Microbiology and Immunology, \\ University of Banja Luka, Banja Luka, Republic of Srpska, Bosnia and Herzegovina \\ ${ }^{2}$ Faculty of Medicine, Institute of Microbiology and Immunology, \\ University of Belgrade, Belgrade, Serbia \\ ${ }^{3}$ Faculty of Medicine, Institute of Medical Statistics and Informatics, \\ University of Belgrade, Belgrade, Serbia
}

(Received: 13 February 2018; accepted: 20 March 2018)

In this study, the focus was on the effects of sub-MICs of the antibiotics on adherence, hydrophobicity, and biofilm formation by two groups of Streptococcus pyogenes strains, which were responsible for different clinical cases. The aim of this study was to explore the effects of sub-MICs of penicillin, ceftriaxone, erythromycin, and clindamycin on adherence, surface hydrophobicity, and biofilm biomass in two selected collections of group A streptococcus (GAS): strains isolated from carriers (CA) and strains isolated from patients with tonsillopharyngitis (TPh). Isolates were tested for hydrophobicity to xylene, adherence, and biofilm production in uncoated microtiter plates before and after treatment with $1 / 2$ and $1 / 4$ MICs of antibiotics. Penicillin reduced adherence and biofilm production in TPh strains, whereas ceftriaxone diminished adherence and biofilm formation in CA group. On the contrary, clindamycin enhanced adherence and biofilm production in both groups of strains. Erythromycin did not significantly alter adherence, but triggered biofilm production in both groups of isolates. Hydrophobicity of both groups of strains was significantly reduced after exposure to all antibiotics. Beta-lactams displayed anti-biofilm activity; penicillin diminished both adherence and biofilm production in TPh strains, whereas ceftriaxone reduced it in strains isolated from CA.

Keywords: Streptococcus pyogenes, adherence, biofilm, antibiotics

*Corresponding author; E-mail: aleksandra.smitran@med.unibl.org 


\section{Introduction}

Streptococcus pyogenes or group A streptococcus (GAS) is one of the most common strictly human pathogens. It causes a wide spectrum of diseases varying from mild, such as impetigo and tonsillopharyngitis (TPh), to life-threatening, such as necrotizing fasciitis and sepsis [1]. In addition, GAS is asymptomatically carried in the throats of healthy persons. A recently conducted meta-analysis estimated the pooled prevalence of GAS carriage among children of all ages at $12 \%$ [2]. The exact mechanisms responsible for this asymptomatic mucosal carriage are not completely understood, but it is well known that patients are likely to have persistent GAS even after administration of the appropriate antimicrobial therapy for TPh. Biofilm formation has been suggested as an important aspect of the carrier (CA) state and antibiotic treatment failures [3]. The ability of GAS to form biofilm was recently recognized as an important virulence trait of the bacterium, and the clinical relevance of GAS biofilm has been corroborated by numerous studies [4-6].

An interesting question is which environmental factors trigger GAS biofilm formation. In addition to the likely candidates, such as oxygen and availability of nutrients $[7,8]$, antibiotics commonly used in the treatment of GAS infections may also affect the capacity of GAS to form biofilm. Numerous studies have shown that antibiotics, when present at concentrations below the minimum inhibitory concentration (MIC), have various effects on bacteria, including morphological changes, altered growth kinetics, changes in adhesive properties, and in synthesis of enzymes and toxins [9, 10]. It has also been shown that biofilm production is among the bacterial traits affected by sub-MICs of antibiotics. While some studies reported inhibitory effects of sub-MICs of antibiotics on biofilm formation [11], several studies carried out over the past decade showed the opposite effect. That is, it has convincingly been shown that sub-MICs of some antibiotics actually induce biofilm formation in different bacteria $[12,13]$.

The studies investigating the interference of sub-MICs of antibiotics with biofilm formation by GAS are scarce and were designed to characterize the antibiofilm activity of fluoroquinolones, the class of antibiotics not used against S. pyogenes $[14,15]$. According to the most recent guidelines of Infectious Diseases Society of America, penicillin remains the treatment of choice for GAS TPh, while patients with penicillin intolerance should be treated with cephalosporins, macrolides, or clindamycin [16]. Antibiotic treatment for the purposes of eradication of GAS carriage is generally not recommended. However, eradication of GAS carriage may be considered under specific circumstances, such as GAS invasive disease outbreaks, and penicillin, macrolides, and clindamycin are recommended options 
$[17,18]$. Therefore, the aim of this study was to explore the role of sub-MICs of penicillin, ceftriaxone, erythromycin, and clindamycin as possible triggers for GAS biofilm production. We assessed the effects of sub-MICs of the four antibiotics on adherence, surface hydrophobicity, and biofilm biomass in two selected collections of GAS strains: strains isolated from CA and strains isolated from patients with TPh.

\section{Materials and Methods}

\section{Bacterial strains}

On the whole, $100 \mathrm{CA}$ and $50 \mathrm{TPh}$ GAS strains were included in the study. All the isolates were collected during 2012, as a part of the national collection of GAS strains formed at the National Reference Laboratory for Streptococci (Faculty of Medicine, Institute of Microbiology and Immunology, University of Belgrade). The GAS isolates were cultured on Columbia agar plates containing $5 \%$ sheep blood and stored at $-80{ }^{\circ} \mathrm{C}$ in Todd Hewitt broth with glycerol (Biolife, Italy). Identification of the strains was based on colony morphology, beta hemolysis, Gram-stain morphology, bacitracin sensitivity, and trimethoprimsulfamethoxazole resistance and was confirmed by the slide agglutination test (Slidex Strepto-Kit, bioMerieux, France).

\section{Quantification of adherence and biofilm production}

Quantification of adherence and biofilm biomass was based upon the protocol described by Stepanovic et al. [19]. The strains were incubated overnight in Todd Hewitt broth with $1 \%$ of yeast extract (THY) at $37^{\circ} \mathrm{C}$, and then diluted in fresh THY medium to achieve final concentration of $10^{6} \mathrm{CFU} / \mathrm{ml}$. Aliquots of bacterial suspension $(100 \mu \mathrm{l})$ were transferred to each well of the 96-well microtiter plate and incubated for $30 \mathrm{~min}$ at $37^{\circ} \mathrm{C}$ for quantification of adherence. The incubation periods for biofilm quantification assays were 12, 24, and $48 \mathrm{~h}$. Following a corresponding incubation period, the content of each well was aspirated, and wells were washed three times with sterile phosphate-buffered saline (PBS). After overnight incubation at room temperature, the plates were stained with $100 \mu \mathrm{l}$ of $2 \%$ (w/v) crystal violet. Afterward, the dye bound to the adherent cells was solubilized with $100 \mu \mathrm{l}$ of $33 \%(\mathrm{v} / \mathrm{v})$ glacial acetic acid. The optical density (OD) of each well was measured at $570 \mathrm{~nm}$, using an automated microtiter plate reader (ICN Flow Titertek Multiskan Plus Reader, Meckenheim, Germany). The negative control wells contained THY broth only. Staphylococcus epidermidis ATCC 14990 was used as the positive control. 
The cut-off $\mathrm{OD}(\mathrm{ODc})$ was defined as three standard deviations above the mean OD of the negative control. The results were interpreted as follows: $\mathrm{OD} \leq \mathrm{ODc}=$ nonadherent isolates/non-biofilm producers, $\mathrm{ODc}<\mathrm{OD} \leq(2 \times \mathrm{ODc})=$ weakly adherent isolates/weak biofilm producers, $(2 \times \mathrm{ODc})<\mathrm{OD} \leq(4 \times \mathrm{ODc})=$ moderately adherent isolates/moderate biofilm producers, and $\mathrm{OD}>(4 \times \mathrm{ODc})=$ strongly adherent isolates/strong biofilm producers. All analyses were performed in triplicate and repeated at least two times.

\section{Assessment of hydrophobicity}

Hydrophobicity was assessed by the microbial adhesion to hydrocarbons test described by Rosenberg et al. [20]. In brief, after overnight incubation in THY, bacteria were harvested by centrifugation and were washed twice in PBS. The pellet was resuspended in PUM buffer [22.2 $\mathrm{g}$ of potassium phosphate trihydrate, $7.26 \mathrm{~g}$ of monobasic potassium phosphate, $1.8 \mathrm{~g}$ of urea, and $0.2 \mathrm{~g}$ of magnesium sulfate heptahydrate/L ( $\mathrm{pH} 7.1)]$ to a standard inoculum corresponding to approximately $10^{8} \mathrm{CFU} / \mathrm{ml}$. An amount of $100 \mu \mathrm{l}$ of xylene was added and the mixture was vigorously vortexed for $1 \mathrm{~min}$, followed by 10-min incubation at ambient temperature to allow separation of the organic and aqueous phases. The $\mathrm{OD}_{540}$ of the lower, aqueous phase was recorded. The percentage of cell surface hydrophobicity, which is a measure of the percentage of bacterial cells separating into the organic phase, was calculated as follows: $\%$ hydrophobicity $=[(\mathrm{A} 0-\mathrm{A} 1) / \mathrm{A} 0] \times 100$, where A0 stands for OD of bacterial suspension measured before adding the hydrocarbon and $\mathrm{A} 1$ for $\mathrm{OD}$ of bacterial suspension measured after adding the hydrocarbon. The assay was performed in triplicate.

Assessment of effects of sub-MICs of antibiotics on adherence, hydrophobicity, and biofilm production

MICs of penicillin, ceftriaxone, erythromycin, and clindamycin (Applichem $\mathrm{GmbH}$, Germany) for all GAS strains tested were determined by broth microdilution method, according to the EUCAST recommendations [21]. To evaluate the effects of sub-MICs of the antibiotics on adherence, hydrophobicity, and biofilm biomass, the first step was overnight incubation of GAS strains in THY with an antibiotic in final concentrations corresponding to $1 / 2$ and $1 / 4$ of its MIC values. The bacterial suspensions for adherence, hydrophobicity, and biofilm testing were also prepared in THY medium with both sub-MICs of an antibiotic. Finally, adherence, hydrophobicity, and biofilm biomass of the GAS strains exposed to sub-MICs of antibiotics were quantified by performing the protocols described above. 
Statistical analysis

Student's $t$-test was used to measure the differences in adherence, hydrophobicity, and biofilm production before and after exposure to an antibiotic within each group of GAS strains tested. Data analyses were carried out using the SPSS version 20. The differences were considered significant if $p<0.05$, and highly significant if $p<0.01$.

\section{Results}

According to the MIC values obtained, all 100 GAS strains were associated with carriage, and 50 strains recovered from patients with $\mathrm{TPh}$ were susceptible to penicillin, ceftriaxone, erythromycin, and clindamycin.

\section{Effects of sub-MICs of antibiotics on adherence of GAS strains}

The proportions of adherent isolates established among GAS strains grown in the absence of an antibiotic compared with those of strains after overnight incubation with 1/2 and 1/4 MICs of each antibiotic are presented in Figure 1. All GAS isolates displaying adherence, both in the absence and the presence of antibiotics, were classified as weakly adherent. Without exposure to antibiotics, proportions of adherent isolates in CA and TPh groups were similar $(p=0.816)$. That is, $53 \%$ of CA and $52 \%$ of TPh isolates were identified as adherent. Exposure to $1 / 2$ and $1 / 4$ MICs of penicillin significantly decreased adherence in TPh strains ( $p=0.001$ and 0.031 , respectively) (Figure 1), while adherence of CA group was not affected. In contrast to the effects of penicillin, both sub-MICs of ceftriaxone reduced adherence in CA group $(p \leq 0.001)$ but not in TPh (Figure 1). Sub-MICs of erythromycin did not significantly alter adherence of GAS strains tested. Clindamycin at both sub-MICs enhanced adherence in TPh strains $(p=0.001$ for $1 / 4 \mathrm{MIC}$ and $p \leq 0.001$ for $1 / 2 \mathrm{MIC}$ ), whereas in CA strains, improved adherence was noted only after exposure to $1 / 2$ MIC of the antibiotic $(p=0.034)$ (Figure 1).

\section{Effects of sub-MICs of antibiotics on hydrophobicity of GAS strains}

$\mathrm{CA}$ and TPh strains cultivated without an antibiotic significantly displayed different surface hydrophobicity (Figure 2). The proportions of hydrophobic isolates in $\mathrm{CA}$ and $\mathrm{TPh}$ groups were $48 \%$ and $23 \%$, respectively $(p \leq 0.001)$. 

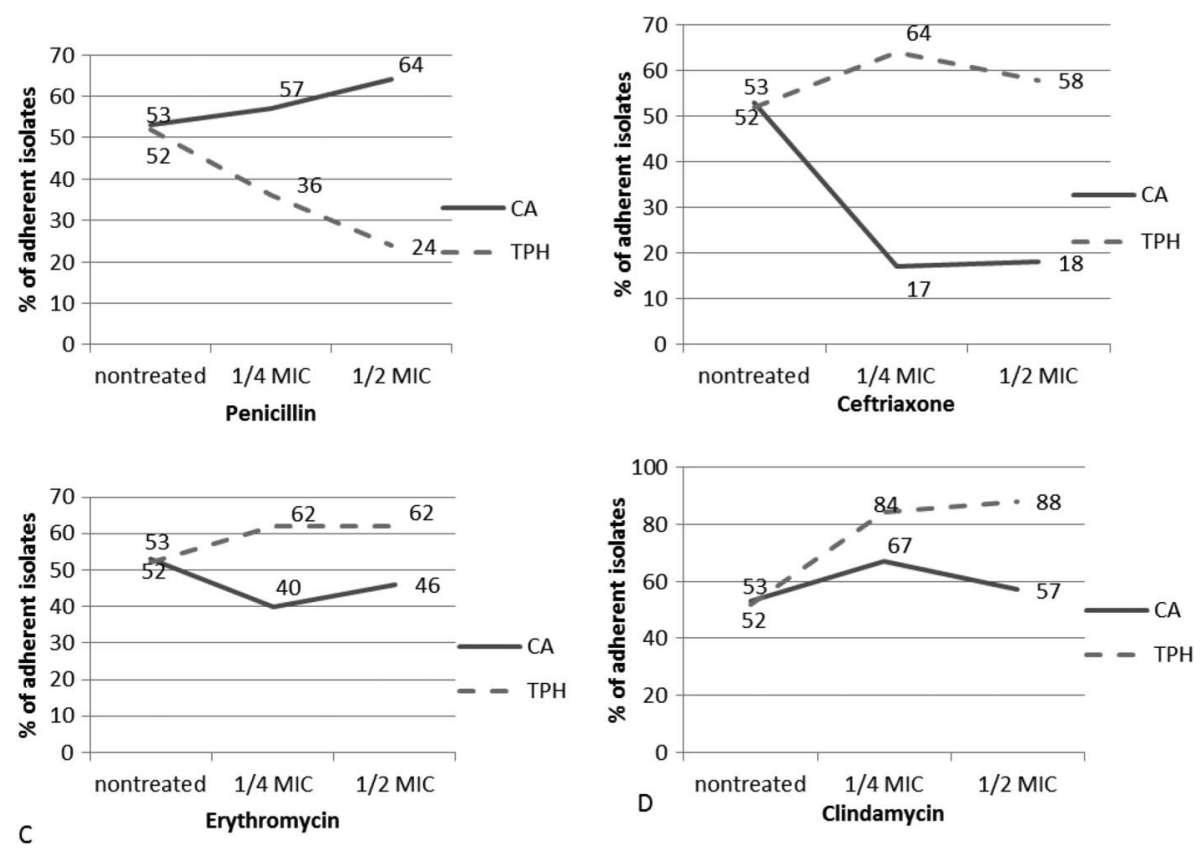

Figure 1. Effects of sub-MICs of antibiotics on adherence of GAS strains. CA: strains isolated from carriers; TPh: strains isolated from patients with tonsillopharyngitis

Hydrophobicity of CA and TPh strains was significantly reduced after exposure to both sub-MICs of penicillin ( $p \leq 0.001)$, ceftriaxone $(p \leq 0.001)$, and clindamycin $(p \leq 0.001)$. Erythromycin exhibited the same effects on hydrophobicity of CA strains $(p \leq 0.001)$, while significant decrease in hydrophobicity of TPh strains was noted only in the presence of $1 / 2$ MIC $(p=0.011)$.

\section{Effects of sub-MICs of antibiotics on biofilm production of GAS strains}

Proportions of biofilm producers among untreated GAS strains and strains exposed to $1 / 2$ and $1 / 4$ MICs of the antibiotics tested during initial overnight incubation and subsequent incubation for 12, 24, and $48 \mathrm{~h}$ are shown in Table I. All untreated isolates identified as biofilm producers displayed low capacity for biofilm formation. The differences in effects of antibiotics on biofilm production by $\mathrm{CA}$ and TPh strains were notable. Penicillin, erythromycin, and clindamycin significantly increased the biofilm capacity of CA strains after 12 and $24 \mathrm{~h}$ of incubation. Ceftriaxone also stimulated biofilm formation after $12 \mathrm{~h}$ of incubation, but prolonged incubation of CA strains with this antibiotic suppressed their ability 

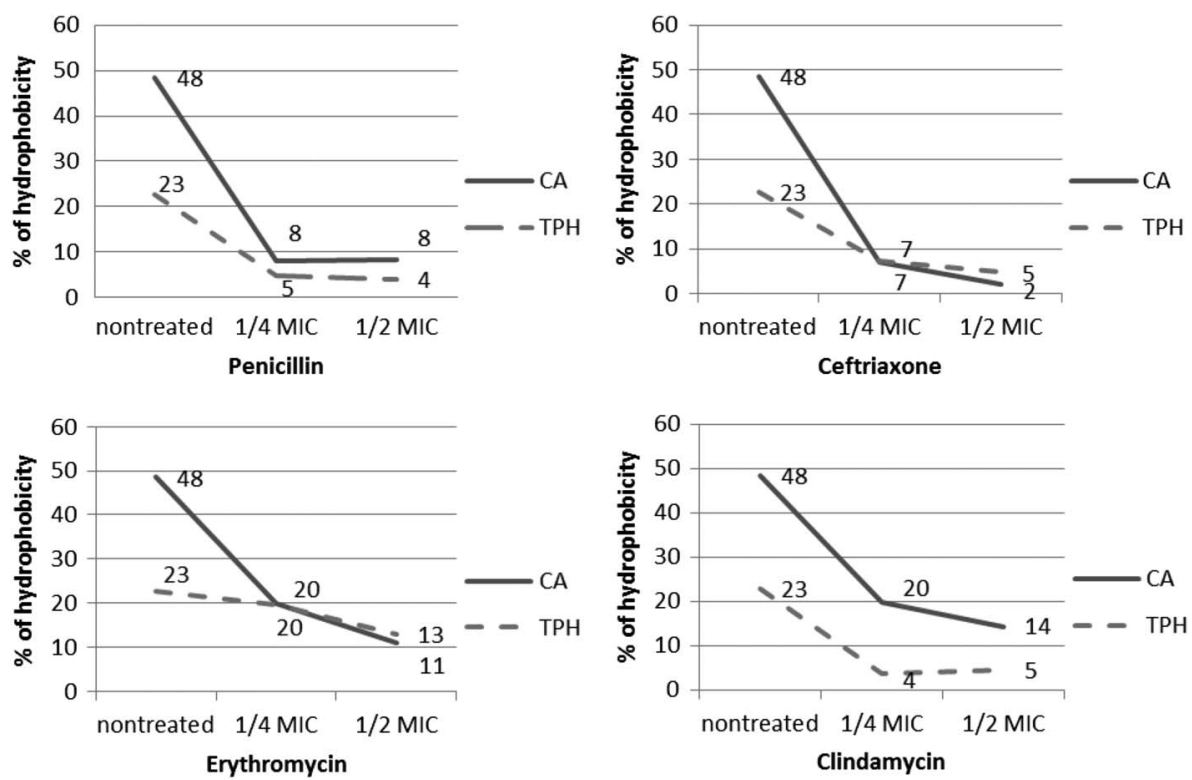

Figure 2. Effects of sub-MICs of antibiotics on hydrophobicity. CA: strains isolated from carriers; $\mathrm{TPh}$ : strains isolated from patients with tonsillopharyngitis

to form biofilm. Both beta-lactams have significantly reduced biofilm formation in $\mathrm{TPh}$ strains, whereas clindamycin and erythromycin at 1/2 MIC had the opposite effects, and triggered biofilm production by these strains.

\section{Discussion}

Since the effects of sub-MICs of antibiotics commonly used against GAS on its biofilm formation have not been studied so far, we investigated $S$. pyogenes biofilm formation in the presence of sub-MICs of the four selected antibiotics by measuring adherence, hydrophobicity, and biofilm biomass. The ability of GAS to adhere to polystyrene plates was studied as the first and fundamental stage of biofilm formation and cell surface hydrophobicity as another important parameter, which influences the initial stages of biofilm formation by promoting cell-substrate interactions [6]. Consistent with the findings of other bacteria [22, 23], we found that sub-MICs of the antibiotics significantly tested modulated biofilm formation by GAS. However, the nature of the modulatory effects, i.e., induction or inhibition of biofilm formation, varied for different antibiotics, and for different groups of strains. 


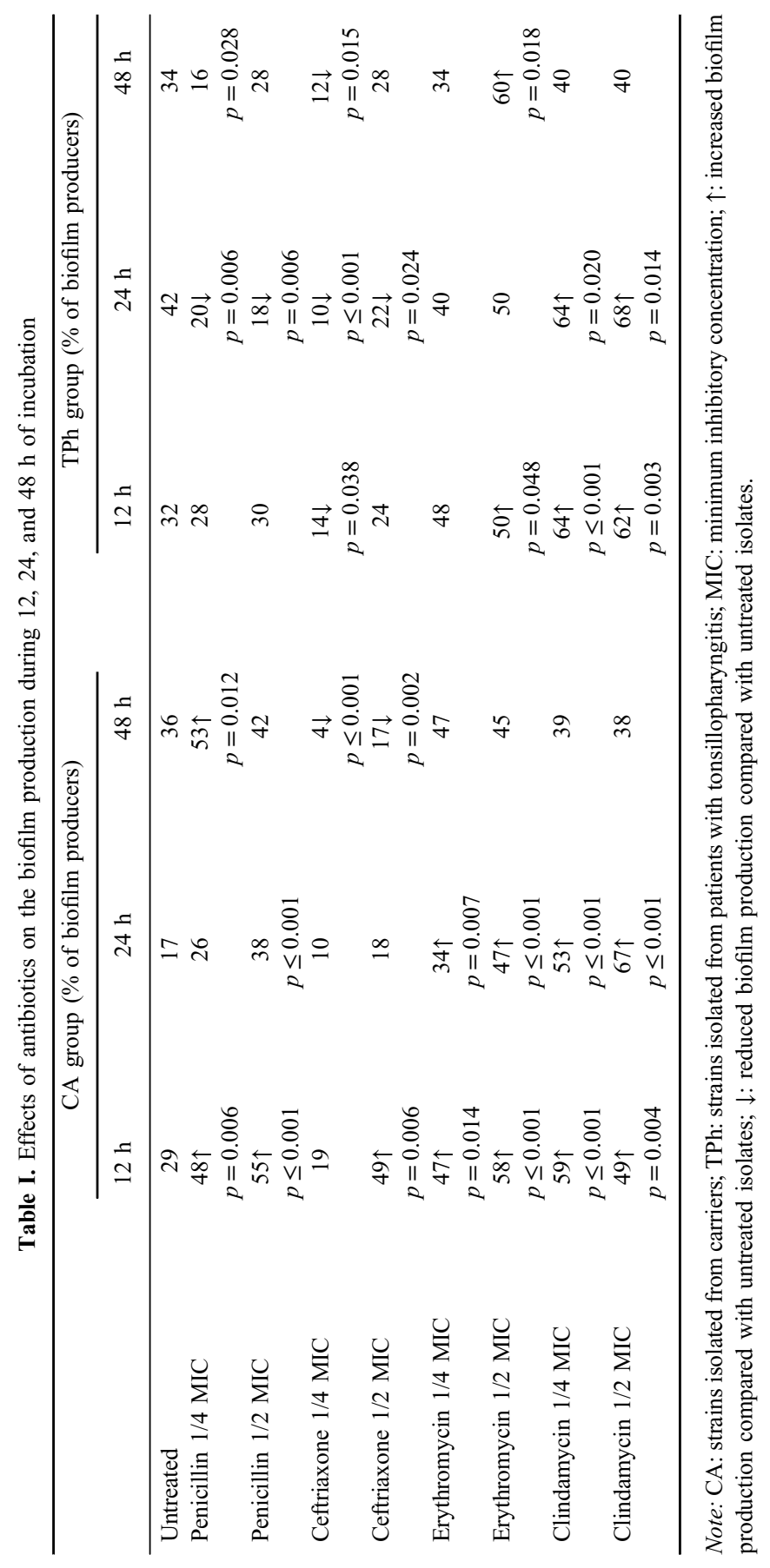


It has already been shown that the sub-MICs of the four antibiotics we tested either enhance or decrease adherence, hydrophobicity, and biofilm formation in bacteria, depending on their species. Sub-MICs of penicillin and erythromycin, for example, increased cell-surface hydrophobicity and stimulated biofilm formation in Corynebacterium diphtheriae [24]. Adherence of uropathogenic Escherichia coli strains, however, was significantly reduced in the presence of penicillin at 1/2 MIC [25]. The same effect on adherence of various Gram-negative and Grampositive bacteria was established for ceftriaxone at 1/4 and 1/8 MICs [26]. In this study, both sub-MICs of penicillin significantly reduced all three parameters, which we quantified in TPh strains and thus showed an apparent anti-biofilm effect. It is noteworthy that penicillin displayed the opposite effect in the CA group of GAS strains and clearly triggered their biofilm production. As far as ceftriaxone is concerned, the overall results obtained indicate excellent inhibitory effects of sub-MICs of this antibiotic on biofilm formation by both CA and TPh GAS isolates. Unlike the anti-biofilm effects of sub-MICs of the two beta-lactam antibiotics tested, our results demonstrated that the sub-MICs of erythromycin and, in particular, clindamycin consistently induced biofilm formation by GAS strains.

Although understanding of the process of biofilm formation by GAS was beyond the scope of this study, the results obtained indicate that alterations in biofilm production caused by sub-MICs of the antibiotics were not directly attributable to the changes in surface hydrophobicity. Sub-MICs of all antibiotics consistently reduced surface hydrophobicity in GAS strains, regardless of their effect on biofilm production. In contrast, relationship between the effects of subMICs of antibiotics on GAS adherence and the effects on biofilm biomass produced was nearly proportional. Reduced adherence caused by sub-MICs of antibiotics was observed in strains, whose biofilm capacity was also negatively affected, while enhancement of biofilm formation by GAS strains coincided with unaltered or increased adherence.

GAS growth in the form of biofilm is presumed to be of critical importance for GAS carriage, recurrent infection episodes, and antibiotic treatment failures [3, 7]. Given the clinical relevance of GAS biofilms, the effects of sub-MICs of the four antibiotics on GAS biofilm formation we observed are intriguing. It is clear that subMICs of antibiotics must be present for certain period of time during and following antibiotic treatments. In this study, the focus was on the effects of sub-MICs of the antibiotics on biofilm formation by two groups of GAS strains, which are responsible for different clinical cases and thus treated differently.

Although we are well aware that converting in vitro observations into in vivo therapeutic regimens is problematical, our major findings support the application of beta-lactams in treatment of TPh. In contrast, sub-MICs of erythromycin, as a 
representative of macrolides, and clindamycin actually triggered biofilm formation by TPh strains. As far as eradication of GAS carriage is concerned, only ceftriaxone at sub-MICs showed promising results regarding inhibition of biofilm formation.

\section{Acknowledgements}

This work was supported by grant no. ON175039 from the Ministry of Education, Science and Technological Development of the Republic of Serbia. The work was performed in the Institute of Microbiology and Immunology, Faculty of Medicine, University of Belgrade.

\section{Conflict of Interest}

The authors have declared that no competing interest exists.

\section{References}

1. Cunningham, M. W.: Pathogenesis of group A streptococcal infection. Clin Microbiol Rev 13, 470-511 (2000).

2. Shaikh, N., Leonard, E., Martin, J. M.: Prevalence of streptococcal pharyngitis and streptococcal carriage in children: A meta-analysis. Pediatrics 126, e557-564 (2010).

3. Ogawa, T., Terao, Y., Okuni, H., Ninomiya, K., Sakata, H., Ikebe, K., Maeda, Y., Kawabata, S.: Biofilm formation or internalization into epithelial cells enable Streptococcus pyogenes to evade antibiotic eradication in patients with pharyngitis. Microb Pathog 51, 58-68 (2011).

4. Roberts, A. L., Connolly, K. L., Kirse, D. J., Evans, A. K., Poehling, K. A., Peters, T. R., Reid, S. D.: Detection of group A streptococcus in tonsils from pediatric patients reveals high rate of asymptomatic streptococcal carriage. BMC Pediatr 12, 3 (2012).

5. Akiyama, H., Morizane, S., Yamasaki, O., Oono, T., Iwatsuki, K.: Assessment of Streptococcus pyogenes microcolony formation in infected skin by confocal laser scanning microscopy. J Dermatol Sci 32, 193-199 (2003).

6. Fiedler, T., Köller, T., Kreikemeyer, B.: Streptococcus pyogenes biofilms-formation, biology, and clinical relevance. Front Cell Infect Microbiol 11, 5-15 (2015).

7. Baldassarri, L., Creti, R., Recchia, S., Imperi, M., Facinelli, B., Giovanetti, E., Pataracchia, M., Alfarone, G., Orefici, G.: Therapeutic failures of antibiotics used to treat macrolidesusceptible Streptococcus pyogenes infections may be due to biofilm formation. J Clin Microbiol 44, 2721-2727 (2006).

8. Thenmozhi, R., Balaji, K., Kumar, R., Rao, T. S., Pandian, S. K.: Characterization of biofilms in different clinical M serotypes of Streptococcus pyogenes. J Basic Microbiol 51, 196-204 (2011). 
9. Andreoni, F., Zürcher, C., Tarnutzer, A., Schilcher, K., Neff, A., Keller, N., Marques Maggio, E., Poyart, C., Schuepbach, R. A., Zinkernagel, A. S.: Clindamycin affects group A streptococcus virulence factors and improves clinical outcome. J Infect Dis 215, 269-277 (2017).

10. Gemmell, C. G., Ford, C. W.: Virulence factor expression by Gram-positive cocci exposed to subinhibitory concentrations of linezolid. J Antimicrob Chemother 50, 665-672 (2002).

11. Wojnicz, D., Tichaczek-Goska, D.: Effect of sub-minimum inhibitory concentrations of ciprofloxacin, amikacin and colistin on biofilm formation and virulence factors of Escherichia coli planktonic and biofilm forms isolated from human urine. Braz J Microbiol 44, 259-265 (2013).

12. Kaplan, J. B., Izano, E. A., Gopal, P., Karwacki, M. T., Kim, S., Bose, J. L., Bayles, K. W., Horswill, A. R.: Low levels of $\beta$-lactam antibiotics induce extracellular DNA release and biofilm formation in Staphylococcus aureus. mBio 3, e00198-12 (2012).

13. Bedran, T. B., Grignon, L., Spolidorio, D. P., Grenier, D.: Subinhibitory concentrations of triclosan promote Streptococcus mutans biofilm formation and adherence to oral epithelial cells. PLoS One 9, e89059 (2014).

14. Balaji, K., Thenmozhi, R., Pandian, S. K.: Effect of subinhibitory concentrations of fluoroquinolones on biofilm production by clinical isolates of Streptococcus pyogenes. Indian J Med Res 137, 963-971 (2013).

15. Shafreen, R. M., Srinivasan, S., Manisankar, P., Pandian, S. K.: Biofilm formation by Streptococcus pyogenes: Modulation of exopolysaccharide by fluoroquinolone derivatives. J Biosci Bioeng 112, 345-350 (2011).

16. Shulman, S. T., Bisno, A. L., Clegg, H. W., Gerber, M. A., Kaplan, E. L., Lee, G., Martin, J. M., Van Beneden, C.: Clinical practice guideline for the diagnosis and management of group A streptococcal pharyngitis: 2012 update by the Infectious Diseases Society of America. Clin Infect Dis 55, 1279-1282 (2012).

17. Walker, M. J., Barnett, T. C., McArthur, J. D., Cole, J. N., Gillen, C. M., Henningham, A., Sriprakash, K. S., Sanderson-Smith, M. L., Nizet, V.: Disease manifestations and pathogenic mechanisms of group A streptococcus. Clin Microbiol Rev 27, 264-301 (2014).

18. Prevention of Invasive Group A Streptococcal Infections Workshop Participants: Prevention of invasive group A streptococcal disease among household contacts of case patients and among postpartum and postsurgical patients: Recommendations from the Centers for Disease Control and Prevention. Clin Infect Dis 35, 950-959 (2002).

19. Stepanovic, S., Vukovic, D., Dakic, I., Savic, B., Svabic-Vlahovic, M.: A modified microtiter-plate test for quantification of staphylococcal biofilm formation. J Microbiol Methods 40, 175-179 (2000).

20. Rosenberg, M., Gutnick, E., Rosenberg, M.: Adherence of bacteria to hydrocarbons: A simple method for measuring cell-surface hydrophobicity. FEMS Microbiol Lett 9, 29-33 (1980).

21. European Committee on Antimicrobial Susceptibility Testing: Breakpoint tables for interpretation of MICs and zone diameters. Version 2.0 (January 2012). Available at http://www.eucast.org/fileadmin/src/media/PDFs/EUCAST_files/Translations/EUCAST_ breakpoints_8.0_srpski.xlsx

22. Kaplan, J. B.: Antibiotic-induced biofilm formation. Int J Artif Organs 34, 737-751 (2011). 
23. Schilcher, K., Andreoni, F., Dengler Haunreiter, V., Seidl, K., Hasse, B., Zinkernagel, A. S.: Modulation of Staphylococcus aureus biofilm matrix by subinhibitory concentrations of clindamycin. Antimicrob Agents Chemother 60, 5957-5967 (2016).

24. Gomes, D. L., Peixoto, R. S., Barbosa, E. A., Napoleão, F., Sabbadini, P. S., dos Santos, K. R., Mattos-Guaraldi, A. L., Hirata, R., Jr.: SubMICs of penicillin and erythromycin enhance biofilm formation and hydrophobicity of Corynebacterium diphtheriae strains. J Med Microbiol 62, 754-760 (2013).

25. Baskin, H., Dogan, Y., Hakki, B. I., Yulug, N.: Effect of subminimal inhibitory concentrations of gentamicin, penicillin, trimethoprim-sulfamethoxazole on adherence of uropathogenic Escherichia coli strains. J Chemother 14, 161-165 (2002).

26. Drago, L., De Vecchi, E., Nicola, L., Tocalli, L., Gismondo, M. R.: Effect of moxifloxacin on bacterial pathogenicity factors in comparison with amoxicillin, clarithromycin and ceftriaxone. J Chemother 16, 30-37 (2004). 